\title{
Dipole binding in a cosmic string background due to quantum anomalies
}

\author{
Pulak Ranjan Gir* \\ Saha Institute of Nuclear Physics, 1/AF Bidhannagar, Calcutta 700064, India
}

(Dated: November 5, 2018)

\begin{abstract}
We propose quantum dynamics for the dipole moving in cosmic string background and show that the classical scale symmetry of a particle moving in cosmic string background is still restored even in the presence of dipole moment of the particle. However, we show that the classical scale symmetry is broken due to inequivalent quantization of the the non-relativistic system. The consequence of this quantum anomaly is the formation of bound state in the interval $\xi \in(-1,1)$. The inequivalent quantization is characterized by a 1-parameter family of self-adjoint extension parameter $\Sigma$. We show that within the interval $\xi \in(-1,1)$, cosmic string with zero radius can bind the dipole and the dipole does not fall into the singularity.

PACS numbers: 03.65.-w, 02.30.Sa, 98.80.Cq, 11.27.+d
\end{abstract}

Anomaly [1, 2] is a breaking of classical symmetry due to quantization of the system, which occurs in various problems in physics. It is one of the three possible symmetry breaking [3] i.e., spontaneous, explicit and anomalous symmetry breaking, which are extremely important due to their consequences in different physical processes. Chiral anomaly [4, 5] is one such important example in high energy physics. In quantum field theory the concept of anomaly has been used successfully [1, 6]. The other important area of physics is string theory [7], where anomaly has also been used successfully. In quantum mechanics, anomaly can be understood as follows. An operator, which is the generator of the symmetry in a classical system, becomes anomalous when it does not keep the domain of the Hamiltonian invariant. By this definition of anomaly, it has been shown that in molecular physics [8] (in quantum mechanical context), there exists interesting scaling anomaly. For example, interaction of an electron in the field of a polar molecule is a simple example of anomaly, where the classical scaling symmetry of the system is broken once it goes inequivalent quantization [9]. An obvious consequence of this scaling anomaly in molecular physics is the occurrence of bound state and the the dependence of momentum in the phase shift of the $S$-matrix.

In cosmic string scenario, scaling anomaly has been observed [11] for particles moving in it, where the induced potential is $1 / r^{2}$ in nature. Inverse square potential appears in various situations in physics starting from molecular physics to black hole [8, 9, 10]. The anomaly, shown in Ref. 11], leads to bound state for the particle. In this letter we consider quantum dynamics of a particle with dipole moment $D$ moving in cosmic string background. This problem has been discussed [12] for large negative coupling constant of the inverse square potential, where the particle falls into the center [13] due to the formation of infinite number of bound states with ground state energy being negative infinite. However fall to the center has been avoided [12] by considering a finite radius for the cosmic string. We will however consider a particular portion of the coupling constant of the inverse square potential which will allow us to obtain nontrivial boundary condition. This nontrivial boundary condition will break the scaling symmetry by introducing a length scale in the form of a single bound state.

Quantum mechanics [14] in cosmic string background has received lot of interest due to its analogy [15] with Aharanov-Bohm effect 16. In relativistic theory it has been shown 17] that Dirac equation in cosmic string background needs nontrivial boundary condition to be imposed on the spinor wave-function at the origin. In language of mathematics the construction of nontrivial boundary condition is usually called self-adjoint extensions [18]. The extensions can be characterized by independent parameters and different values of the parameters lead to inequivalent theories. It has been observed [19] that in cosmic string scenario the fermionic charge can be non-integral multiple of Higgs charge. Since the flux is quantized with respect to the Higgs charge it will lead to nontrivial Aharanov-Bohm scattering for fermion. The cross section increases due to this Aharanov-Bohm scattering in addition to gravitational scattering. In nonrelativistic theory [20], the consideration of inequivalent quantization is also inevitable in order to get bound state for the particle moving in cosmic string background. In Ref. 21] gravitational scattering by particles of a spinning source in two dimensions has been studied. There it has been shown that the energy eigenvalue and corresponding eigenfunction of a particle in the field of a massless spinning source is equivalent to that in a background Aharanov-Bohm gauge field of an infinitely thin flux tube. This topological defect appears in astrophysics 22] and also in condensed matter physics 23].

This letter has been organized in the following way: First, we study the scaling symmetry of the classical system, which undergoes anomalous breaking upon quantization; Second, we make an inequivalent quantization of the system, which is responsible for anomaly and discuss its consequences.

First, Scaling symmetry is associated with the trans- 


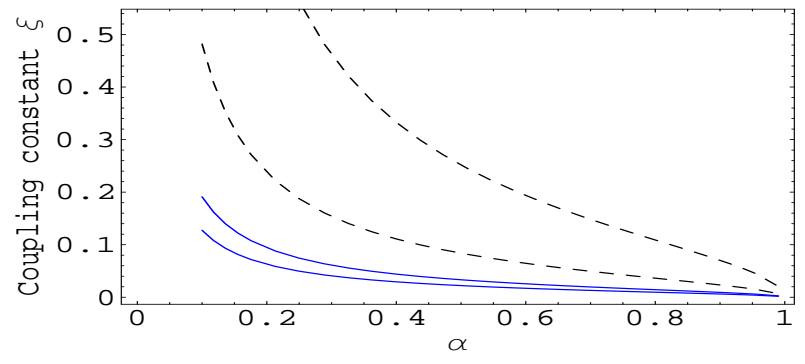

FIG. 1: (color online) A plot of the coupling constant $\xi$ as a function of the cosmic string parameter $\alpha$. Dotted graphs correspond to $\Theta=\pi / 8$ and from top to bottom $D=0.5,1.5$ respectively. Solid graphs (blue) correspond to $\Theta=\pi / 5$ and from top to bottom $D=0.2,0.3$ respectively.

formation $\mathbf{r} \rightarrow \lambda \mathbf{r}$ and $t \rightarrow \lambda^{2} t$, where $\lambda$ is the scaling factor. In classical physics when the action is invariant under this transformation, then the corresponding system has scale symmetry. Since in non-relativistic quantum theory, cosmic string induces a $V=\frac{\left(1-\alpha^{2}\right) D^{2}}{48 \pi \alpha^{2} r^{2}} \cos 2 \Theta[24]$ potential to the the particle with dipole moment $D$ moving in its background, the relevant classical symmetry would be the scale symmetry. To be more specific classically, the dipole moving in cosmic string background can be described by the Lagrangian $L=\frac{M}{2} g_{i j} \dot{\mathbf{r}}^{i} \dot{\mathbf{r}}^{j}-V$. This Lagrangian $L$ scales as $\frac{1}{\lambda^{2}} L$. So the action $\mathcal{A}=\int d t L$ will be scale invariant under the transformation $\mathbf{r} \rightarrow \lambda \mathbf{r}$ and $t \rightarrow \lambda^{2} t$. The scale invariance of this action means, if $\psi$ is an eigenstate of the Hamiltonian $H=\frac{M}{2} g_{i j} \dot{\mathbf{r}}^{i} \dot{\mathbf{r}}^{j}+V$ with eigenvalue $E$, i.e., $H \psi=E \psi$, then $\psi_{\lambda}=\psi(\lambda \mathbf{r})$ will also be an eigenstate of the same Hamiltonian with energy $E / \lambda^{2}$. This essentially means that the system with scale symmetry does not have any lower bound in energy and therefore cannot have any bound state. Scale symmetry is however a part of larger conformal symmetry formed by three generators: the Hamiltonian $H$, the Dilatation generator $\mathcal{D}=t H-\frac{1}{4}(\mathbf{r} . \mathbf{p}+\mathbf{p . r})$ and the conformal generator $K=H t^{2}-\frac{1}{2}(\mathbf{r} \cdot \mathbf{p}+\mathbf{p . r})+\frac{1}{2} M \mathbf{r}^{2}$. They form the $S O(2,1)$ algebra: $[\mathcal{D}, H]=-i \hbar H,[\mathcal{D}, K]=i \hbar K,[H, K]=2 i \hbar \mathcal{D}$ [25]. We will show in our case that this scale symmetry will break once the the classical system is quantized.

We consider a non-relativistic particle of mass $M$, dipole moment $D$ moving in the background field of cosmic string. The background is described by the spacetime metric in cylindrical coordinate $(r, \phi, z)$ as

$$
d s^{2}=d t^{2}-d z^{2}-d r^{2}-\alpha^{2} r^{2} d \phi^{2},
$$

where $\alpha=1-4 G \mu<1$ characterizes the string, with $\mu$ is the mass per unit length of the string. The constant $\alpha$ introduces an angular deficit of $2 \pi(1-\alpha)$ in the Minkowski space-time. The interaction between the dipole and the cosmic string background is described by the electromagnetic self-energy 24] of the dipole due to the non-flat geometry. The potential induced in the non-relativistic system is $V=\frac{\left(1-\alpha^{2}\right) D^{2}}{48 \pi \alpha^{2} r^{2}} \cos 2 \Theta[24]$, where $\Theta$ is the angle

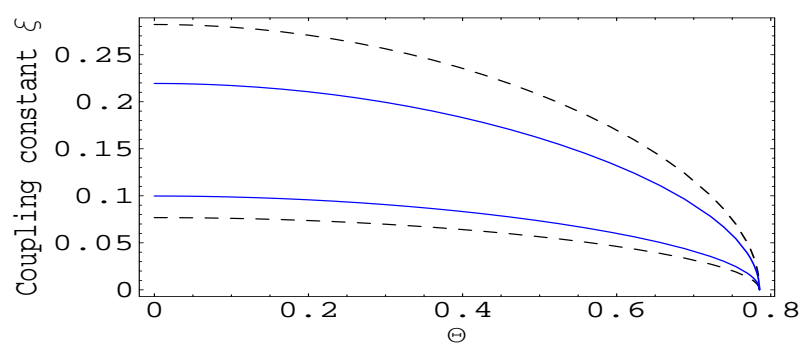

FIG. 2: (color online) A plot of the coupling constant $\xi$ as a function of $\Theta$. Dotted graphs correspond to $D=0.5$ and from top to bottom $\alpha=0.2,0.6$ respectively. Solid graphs (blue) correspond to $\alpha=0.5$ and from top to bottom $D=0.5,1.1$ respectively.

between the string and the dipole moment. This potential transforms under the scale transformation $\mathbf{r}=\lambda \mathbf{r}$ and $t=\lambda^{2} t$ in such a way that the Schrödinger equation for the system becomes scale covariant. Due to cylindrical symmetry of the space, we can easily see that the motion of the particle in the $\mathrm{z}$ direction is basically a free particle motion, described by the wave-function $e^{i k z}$, where $k$ is wave-vector of the particle along the $z$ direction. Since we are considering an infinite cosmic string along the $z$ direction, it is enough to discuss the motion of the particle on the plane perpendicular to the $z$ direction. The motion of the particle on the plane perpendicular to the $z$ axis is described by the time independent Schrödinger equation (in $\hbar^{2}=M=1$ unit)

$$
\left(-\frac{1}{2} \nabla^{2}+\frac{\left(1-\alpha^{2}\right) D^{2}}{48 \pi \alpha^{2} r^{2}} \cos 2 \Theta\right) \Psi=E \Psi
$$

The wave-function can be separated as $\Psi(r, \phi)=$ $R(r) \exp (i m \phi)$ and (2) gives the radial equation

$$
H_{D} R(r) \equiv-\left(\frac{d^{2}}{d r^{2}}+\frac{1}{r} \frac{d}{d r}-\frac{\xi^{2}}{r^{2}}\right) R(r)=2 E R(r),
$$

where $H_{D}$ is the radial Hamiltonian, with $\xi^{2}=$ $\frac{1}{\alpha^{2}}\left(\frac{\left(1-\alpha^{2}\right) D^{2}}{24 \pi} \cos 2 \Theta-m^{2}\right)$ and $m=0, \pm 1, \pm 2, \cdots$. We will now discuss the solution of the Hamiltonian $H_{D}$.

To discuss that we need to know some general properties of an operator, let say $A$. In this article, let us restrict ourselves to the case of unbounded operator, because the Hamiltonian we are discussing is unbounded. Now, for an unbounded operator $A$, one can define a domain $D(A)$, such that the domain is dense in the Hilbert space. From the information of $A$ and $D(A)$, one can construct the adjoint operator $A^{*}$ and the corresponding domain $D\left(A^{*}\right)$ by using the relation $\int_{0}^{\infty} \phi^{*}(r) A \chi(r) d r=\int_{0}^{\infty}\left(A^{*} \phi(r)\right)^{*} \chi(r) d r, \forall \chi(r) \in$ $D(A)$. The condition for self-adjointness for the operator $A$ is given by $D(A)=D\left(A^{*}\right)$. An alternative definition of self-adjointness is given in terms of deficiency indices, found by using von Neumann's method. According to von Neumann's method, the deficiency indices $n_{ \pm}$ 


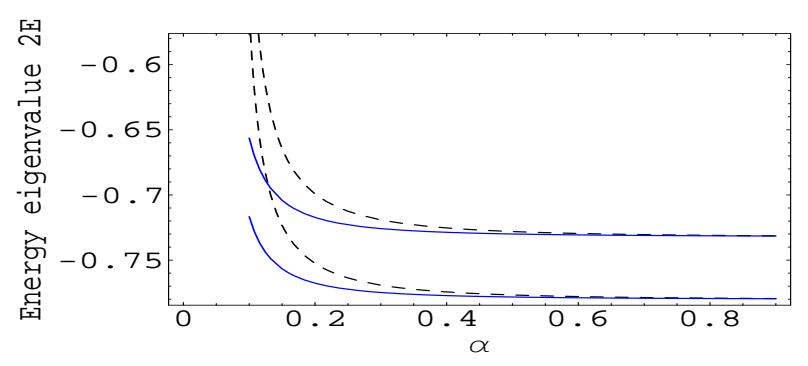

FIG. 3: (color online) A plot of the energy eigenvalue $2 E$ as a function of the cosmic string parameter $\alpha$. Dotted graphs correspond to $\Theta=\pi / 8, D=1.6$ and from top to bottom $\Sigma=\pi / 8, \pi / 10$ respectively. Solid graphs (blue) correspond to $\Theta=\pi / 12, D=1$ and from top to bottom $\Sigma=\pi / 8, \pi / 10$ respectively.

are defined by dimension of the kernel $\operatorname{Ker}\left(i \pm A^{*}\right)$. If $n_{ \pm}=0$, then the operator $A$ is essentially self-adjoint. If $n_{+}=n_{-}=n \neq 0$, then $A$ is not self-adjoint but admits self-adjoint extensions. Self-adjoint extensions can be characterized by $n^{2}$ parameters. Different values of the parameters give rise to different physics. For, $n_{+} \neq n_{-}$, the operator $A$ cannot have any self-adjoint extensions.

Let us now come back to the discussion our operator of interest, which is $H_{D}$. The Hamiltonian $H_{D}$ acts over the Hilbert space of square-integrable functions, given by the domain $\mathcal{L}^{2}\left[R^{+}, r d r\right]$. As discussed earlier, classically this system is scale invariant under the scale transformation $\mathbf{r}=\lambda \mathbf{r}, t=\lambda^{2} t$. It can also be understood from the fact that the coupling constant of the inverse square potential $\xi$ is dimensionless coefficient. Now, we need to see whether this scale symmetry is still restored after quantization. Since this kind of model (inverse square interaction) has been investigated extensively in literature, we know that the Hamiltonian $H_{D}$ is essentially self-adjoint for $\xi^{2} \geq 1$. Since any system is defined by a Hamiltonian and its corresponding domain, in our case the Hamiltonian $H_{D}$ for $\xi^{2} \geq 1$ acts over the domain

$$
\mathcal{D}_{0}=\left\{\psi \in \mathcal{L}^{2}(r d r), \psi(0)=\psi^{\prime}(0)=0\right\}
$$

Let us now investigate another portion of the coupling constant $\xi^{2}$. In this article we will not consider the strong region, because it has been investigated earlier. So the remaining region left to be investigated is $\xi \in(-1,1)$. In this region the Hamiltonian is not essentially self-adjoint and therefore we need to make self-adjoint extensions of the original domain, so that the Hamiltonian becomes self-adjoint. For the moment we consider $\xi \neq 0$, because the case $\xi=0$ should be treated separately. From now onward we confine our analysis to the zero angular momentum states, i.e., we set $m=0$ in the expression of $\xi$ for simplicity. However our analysis is valid as long as $0 \leq \xi^{2}<1$. Since the quantum dynamics in the interval $\xi \in(-1,1)$ essentially depends on the behavior of the coupling constant $\xi$, we plot $\xi$ as a function of the cosmic string parameter $\alpha$ in FIG. 1 and in FIG. 2 , we plot the same thing as a function of the variable $\Theta$. Note that for $\xi \in(-1,1)$, the deficiency indices are $(1,1)$, so the self-adjoint extensions are characterized by a 1-parameter element $e^{i \Sigma}$. Now, the domain under which our Hamiltonian $H_{D}$ should be self-adjoint is given by $\mathcal{D}_{\Sigma}$. Mathematically this domain can be represented by

$$
\mathcal{D}_{\Sigma}=\left\{\mathcal{D}_{0}+\phi_{+}+e^{i \Sigma} \phi_{-}\right\},
$$

where the deficiency space solutions $\phi_{ \pm}$are

$$
\phi_{+}=K_{\xi}\left(r e^{-i \pi / 4}\right), \quad \phi_{-}=K_{\xi}\left(r e^{+i \pi / 4}\right),
$$

where $K_{\xi}$ is the modified Bessel function [26]. The behavior of any function, belonging to the domain $\mathcal{D}_{\Sigma}$, near singularity $r \rightarrow 0$ can be found from the behavior of $\phi_{+}+e^{i \Sigma} \phi_{-}$at short distance, because near singularity, functions belonging to the domain $\mathcal{D}_{0}$ goes to zero. Therefore

$$
\phi_{+}+e^{i \Sigma} \phi_{-} \simeq \mathcal{A}_{+}\left(\frac{r}{2}\right)^{\xi}+\mathcal{A}_{-}\left(\frac{2}{r}\right)^{\xi}
$$

where, $\mathcal{A}_{ \pm}=-\frac{\pi i}{\sin (\pi \xi)} \frac{\cos \left(\frac{\Sigma}{2} \pm \frac{\pi \xi}{4}\right)}{\Gamma(1 \pm \xi)}$.

Let us now solve the eigenvalue problem (3). Since for $\xi^{2} \geq 1$, the Schrödinger equation does not have any normalizable solutions, we can't have any bound state, because bound state solutions must be normalized in quantum mechanics. On the other hand it can be shown that [10] for $\xi \in(-1,1)$, there is exactly one bound state with energy $2 E$, and eigenfunction $R(r)$ :

$$
2 E=-\sqrt[\xi]{\frac{\cos \frac{1}{4}(2 \Sigma+\xi \pi)}{\cos \frac{1}{4}(2 \Sigma-\xi \pi)}}, \quad R(r)=K_{\xi}(\sqrt{2 E} r)
$$

The bound state energy $2 E$ in (8) as a function of the cosmic string parameter $\alpha$ has been plotted in FIG. 3. In FIG. 4, the eigenvalue $2 E$ has been plotted as a function of the dipole moment $D$.

As pointed out before, according to scale symmetry there should not have any bound state solution. But in our system we get bound state solution for nontrivial boundary condition. The bound state eigenvalue can be considered as a scale in the system, which has emerged due to nontrivial boundary condition. Thus the classical scale symmetry is destroyed after quantization of the system. Let us now discuss the scaling anomaly in terms of operators over the Hilbert space. In quantum mechanics there is a operator called scaling operator, which encodes the features of scaling symmetry. The scaling operator is given by

$$
\Lambda=\frac{1}{2}(r p+p r), \quad \text { where } p=-i \frac{d}{d r}
$$

One can check that this scaling operator $\Lambda$ is symmetric on the domain $\mathcal{D}_{0}$ of the Hamiltonian $H_{D}$. It can also 


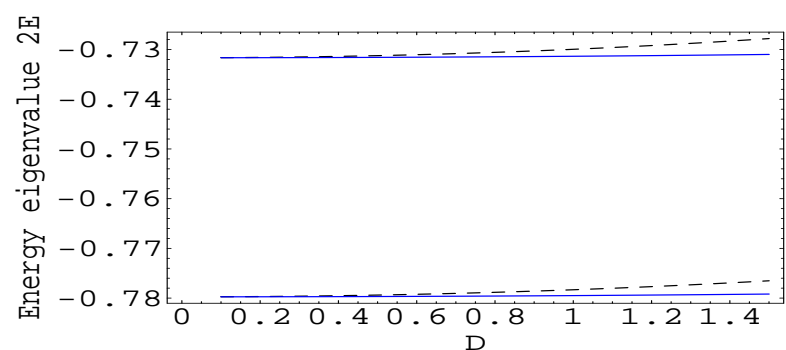

FIG. 4: (color online) A plot of the energy eigenvalue $2 E$ as a function of the dipole moment $D$. Solid graphs (blue) correspond to $\Theta=\pi / 10, \alpha=0.8$ and from top to bottom $\Sigma=\pi / 8, \pi / 10$ respectively. Dotted graphs correspond to $\Theta=\pi / 12, \alpha=0.5$ and from top to bottom $\Sigma=\pi / 8, \pi / 10$ respectively.

be checked that for $\xi^{2} \geq 1$, the domain of the Hamiltonian $H_{D}$ remains invariant when $\Lambda$ when acts on it. For $\xi^{2} \in(-1,1), \Lambda \phi=-\frac{i}{2}\left(\phi+2 r \phi^{\prime}\right)$, where $\phi$ is any element, belonging to the domain $\mathcal{D}_{\Sigma}$. The behavior of the function $\Lambda \phi$ near singularity $(r \rightarrow 0)$ can be found as

$$
\Lambda \phi \simeq-\frac{i}{2}\left[(1+2 \xi) \mathcal{A}_{+}\left(\frac{r}{2}\right)^{\xi}+(1-2 \xi) \mathcal{A}_{-}\left(\frac{2}{r}\right)^{\xi}\right]
$$

where, the constants $\mathcal{A}_{ \pm}$are defined above.

Comparing the expression (5) and (10), we see that $\Lambda \phi$ does not leave the domain of the Hamiltonian invariant, due to the two different terms $(1+2 \xi)$ and $(1+2 \xi)$ in the expression (10). Scaling symmetry is thus broken anomalously. The reason for this anomaly is the inequivalent quantization of the system, by making a self-adjoint extensions of the initially non self-adjoint Hamiltonian. Note that not all values of the self-adjoint extensions parameter $\Sigma$ give rise to scaling anomaly. There are some values of the parameters for which scale symmetry is restored even after quantization. For example, for $\Sigma=\left(1 \pm \frac{\xi}{2}\right) \pi$ there is no bound state. One can also check from (10) that for $\Sigma=\left(1 \pm \frac{\xi}{2}\right) \pi, \Lambda$ leaves the domain of the Hamiltonian invariant.

The case for $\xi=0$ can be handled in a similar fashion. The bound state energy and the wave function in this case are given by

$$
2 E=-\exp \left[\frac{\pi}{2} \cot \frac{\Sigma}{2}\right], \quad R(r)=K_{0}(\sqrt{-2 E} r) .
$$

respectively, where $K_{0}[26]$ is the modified Bessel function. Here also the existence of bound state imply breaking of scale symmetry.

In conclusion, we have shown that the presence of dipole moment of a particle, moving in cosmic string background, does not break the classical scale symmetry, which was present without the dipole moment. However, scale symmetry is anomalously broken by the inequivalent quantization of the system. The inequivalent quan- tization is characterized by one parameter family of selfadjoint extensions. The consequence of this anomaly is the existence of bound state for the dipole and the scale is provided by the bound state energy. We have shown that scale symmetry can be restored for $\Sigma=\left(1 \pm \frac{\xi}{2}\right) \pi$ even after quantization.

* Electronic address: pulakranjan.giri@saha.ac.in

[1] J. F. Donoghue, E. Golowich and B. R. Holstein, Dynamics of the Standard Model (Cambridge University Press, Cambridge, U. K., 1992).

[2] S. B. Treiman, R. Jackiw, B. Zumino and E. Witten, Current Algebra and Anomalies (World Scientific, Singapore, 1985).

[3] M. E. Peskin and D. V. Schroeder, An Introduction to Quantum Field Theory (Westview Books,1993).

[4] J. S. Bell and R. Jackiw, Nuove Cimento A60, 47 (1969); R. Jackiw, Lectures on Current Algebra and its Applications (Princeton University Press, Princeton, 1972).

[5] S. Adler, Phys. Rev 177, 2426 (1969).

[6] L. N. Epele, C. A. García Canal and W. A. Ponce. Phys. Lett. B411, 159 (1997).

[7] J. Polchinski, String Theory (Cambridge University Press, Cambridge, U.K., 1998), Vol I and II.

[8] H. E. Camblong, L. N. Epele, H. Fanchiotti and C. A. G. Canal, Phys. Rev. Lett. 87220402 (2001); H. E. Camblong, C. R. Ordonez, Phys. Rev. D68, 125013 (2003).

[9] P. R. Giri, K. S. Gupta, S. Meljanac and A. Samsarov, hep-th/0703121v1; E. Gerjuoy, Phys. Today 18 24 (1965); J. E. Turner, Phys. Rev. 14121 (1966); G. S. Hurst, L. B. O'Kelly and J. A. Stokdale, Nature 195 66 (1962); G. S. Hurst, J. A. Stokdale and L. B. O'kelly, J. Chem. Phys. 382572 (1963); S. Altshular, Phys. Rev. 107114 (1957); M. H. Mittleman and R. E. von Holdt, Phys. Rev. 140 A726 (1965); K. Fox and J. E. Turner, Am. J. Phys. 34606 1966; K. Fox and J. E. Turner, J. Chem. Phys. 451142 (1966); J. M. Lévy-Leblond, Phys. Rev. 1531 (1967).

[10] B. Basu-Mallick and Kumar S. Gupta, Phys. Lett. A292, 36 (2001); T. R. Govindarajan, V. suneeta and S. Vaidya, Nucl. Phys. B583, 291 (2000); B. Basu-Mallick, Pijush K. Ghosh and Kumar S. Gupta, Nucl. Phys. B659, 437 (2003); Phys. Lett. A311, 87 (2003); Kumar S. Gupta, Mod. Phys. Lett. A18, 2355 (2003); D. Birmingham, Kumar S. Gupta and Siddhartha Sen, Phys. Lett. B505, 191 (2001); Kumar S. Gupta and Siddhartha Sen, Phys. Lett. B526, 121 (2002); S. Meljanac, A. Samsarov, B. Basu-Mallick and Kumar S. Gupta, Eur. Phys. J. C49, 875 (2007).

[11] P. R. Giri, arXiv:0704.1725v2 [hep-th].

[12] C. A. de L. Ribeiro, C. Furtado and F. Moraes, Mod. Phys. Lett. A20, 1991 (2005); C. A. de L. Ribeiro, C. Furtado, V. B. Bezerra and F. Moraes, J. Phys. A34, 6119 (2001).

[13] L. D. Landau and E. M. Lifshitz, Quantum mechanics (Pergamon Press, London, 1959);

[14] A. L. C. de Oliveira and E. R. B. de Mello, Class. Quantum Grav. 22, 1255 (2005); J. L. A. Coelho and R. L. P. G. Amaral, J. Phys. A35, 5255 (2002). 
[15] M. Peskin, Phys. Rev. A23, 360 (1981); V. B. Bezerra, J. Math. Phys. 38, 2553 (1997); C. Furtado, V. B. Bezerra, F. moraes, Mod. Phys. ett. A15, 253 (2000).

[16] Y. Aharanov and D. Bohm, Phys. Rev. 119, 485 (1959).

[17] Ph. de Sousa Gerbert, Phys. Rev. D40, 1346 (1989); B. S. Kay and U. M. Studer, Commun. Math.Phys. 139, 103 (1991).

[18] M. Reed and B. Simon, Fourier Analysis, SelfAdjointness ( New York :Academic, 1975 ); Dunford and J. T. Schwartz, Linear Operators, Spectral Theory, Self Adjoint Operators in Hilbert Space, Part 2 (WileyInterscience; Wiley Clas edition, 1988).

[19] M. Alford and F. wilczek, Phys. Rev. Lett. 62, 1071 (1989).

[20] C. Filgueiras and F. Moraes, Phys. Lett. A361, (2007).

[21] Ph. de Sousa Gerbert and R. Jackiw, Commun. Math. Phys. 124, 229 (1989).

[22] P. Bhattacharjee, Phys. Rev. Lett. 81, 260 (1998); P.
Bhattacharjee, N. Sahu and U. A. Yajnik, Phys.Rev. D70, 083534 (2004); Nucl.Phys. B752, 280-296 (2006).

[23] S. Azevedo, F. Moraes, Phys. Lett. A246, 374 (1998);

S. Azevedo, J. Pereira, Phys. Lett. A275, 463 (2000);

S. R. Vieira, S. Azevedo, Phys. Lett. A288, 29 (2001);

S. Azevedo, Phys. Lett. A293, 283 (2002); R. Ray and A. M. Srivastava, Phys.Rev. D69, 103525 (2004); S. Digal, R. Ray and S. Sengupta, A. M. Srivastava, Phys. Rev. Lett. 84, 826 (2000); S. Digal, R. Ray and A. M. Srivastava, Phys. Rev. Lett. 83, 5030 (1999).

[24] Y. grats and A. garcia, Class. Quant. Grav. 13, 189 (1996).

[25] B. Wybourne, Classical Groups for Physics (Wiley, New York, 1974).

[26] M. Abromowitz, I. A. Stegun, Handbook of Mathematical Functions (Dover, New York, 1970). 